\title{
EVALUATING VISUALIZATION FOR SLIDE-BASED INVESTIGATIVE LEARNING WITH CONNECTION BETWEEN PRESENTATION SLIDES
}

\author{
Mitsuhiro Goto and Akihiro Kashihara \\ The University of Electro-Communications \\ 1-5-1, Chofugaoka, Chofu, 182-8585, Tokyo, Japan
}

\begin{abstract}
Presentation documents have been increasingly used not only as supplementary presentation materials but also as contents for investigative learning. In learning a presentation document, learners generally need to select a number of slides from the document, which describe what they want to learn or should learn. However, it is not so easy to find out the slides to be learnt since what each slide represents is not concisely stated and what connections exist between the adjacent slides are not explicitly expressed. They accordingly take more time for learning and finish learning with incomplete knowledge. In order to address this issue, this paper introduces a map called slidemap for visualizing the logical connections between the slides. This map helps learners identify the sequence of slides to be learnt from the presentation document and grasp the structure embedded in the document in a shorter time. This paper also demonstrates a tool for presentation slides-based investigative learning with slidemap. In addition, this paper reports a case study involving 16 participants with the tool, whose purpose was to ascertain whether using slidemap could be more beneficial for understanding the presentation document than using PowerPoint user interface. The results of the study show the effect of increasing learning time and visiting frequency for slides to be learnt, and promoting learners' understanding of presentation documents in a shorter time.
\end{abstract}

\section{KEYWORDS}

Presentation Slides, Slides-Based Investigative Learning, Visualization, Slide Map

\section{INTRODUCTION}

In recent years, the opportunities of investigative learning with presentation documents (referred to as "P-documents") have been increased since the Web services such as SlideShare (LinkedIn, 2006) allow the learners to search and investigate P-documents in various fields to learn. Corbeil states "For university students familiar to ICT, P-documents are more effective learning media than textbooks (Crebeil, 2007)." In addition, P-documents usually consist of slides, and the contents of a presentation are often summarized clearly and precisely on the slides utilizing figures or images so that the audience can easily comprehend the contents. By using such P-documents, it is possible to learn about the latest research with texts, figures or images in the slides, although those do not exhaustively describe it. For example, researchers in educational technology can learn the contents of slides describing "evaluation methods" from a P-document on research similar to their theme in order to understand "the latest evaluation methods to claim the usefulness of the system." We call such learning with P-documents "slide-based investigative learning (SBI-learning)". Web-based investigative learning has been proposed in our previous work (Kashihara \& Akiyama, 2013). In SBI-learning, on the other hand, learners learn the slides of P-documents instead of web resources, in which they do not always investigate all of the slides included in a P-document, but select a number of slides according to the viewpoint from which they want to learn. They then extract concepts included in slides as keywords to combine them and construct knowledge. Even if the P-document does not contain enough information for learning, they could explore other P-documents related to the viewpoint with the Web services such as SlideShare.

In this work, we have designed a model of SBI-learning that consists of the following three phases: (1) slides investigation phase, (2) knowledge construction phase, and (3) P-documents investigation phase. We have also proposed the method for supporting SBI-learning based on this model. In this paper, we focus on 
slides investigation phase, and introduce a supporting method in this phase. P-documents targeted in this model have a relatively large number of slides (several dozen or so), and can be divided into several semantic segments that embody the main topic of presentations (Shibata et al., 2013), such as sub-themes and topics. Each segment also contains a series of one or more slides, which are logically connected to each other. Furthermore, the slides can be classified into two according to their roles, such as "slides describing the main topic of a presentation" and "slides supplementing the main topic".

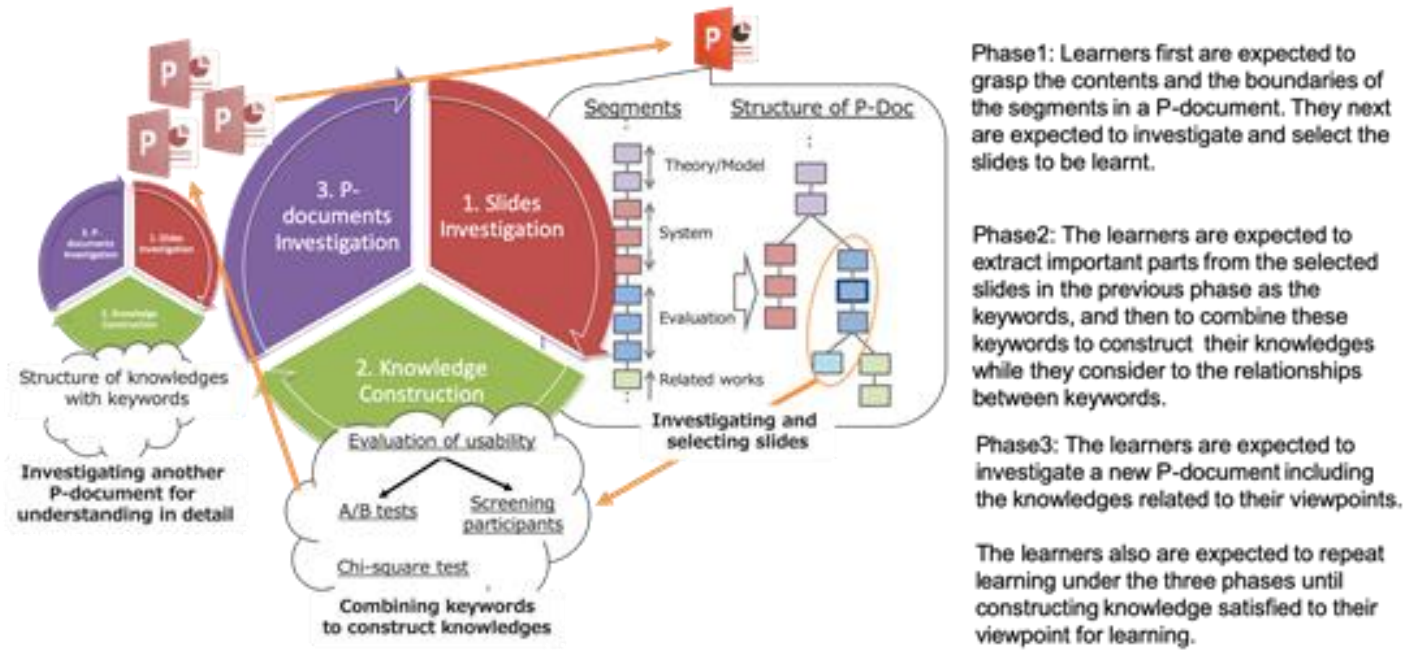

Figure 1. An overview of SBI-learning model

In order to efficiently find out slides to be learnt in a P-document, it is necessary to grasp the contents and boundaries of the segments in the document, and to select the slides based on the segments grasped. However, it is not so easy for learners to identify the segments and to find out slides from P-documents since the roles of slides or the logical connections between the adjacent slides are not explicitly represented. As a result, they take more time for learning, and finish learning with incomplete knowledge.

In this work, we propose a map that visualizes the structure of a P-document involving the roles of each slide and the logical connections between the adjacent slides. This map makes it easy to identify the contents and boundaries of segments in a P-document and to find out slides to be learnt. We also implemented a tool for investigating slides based on the map.

\section{SLIDE-BASED INVESTIGATIVE LEARNING}

\subsection{Overview}

By using P-documents for technical reports, conferences, etc., learners can learn the latest content that is not published in textbooks. During SBI-learning, the learners are expected to investigate a number of slides, which are related to their viewpoints from which they want to learn, and to extract important parts from the slides as keywords that are necessary for learning. They are then expected to combine these keywords to construct their knowledge. Unlike textbooks, these P-documents do not often provide a table of the contents with which the learners can readily detect a number of slides to be learnt and decide the order of learning the slides. Therefore, the learners need to grasp the contents and boundaries of the segments in a P-document considering the roles of the slides and the logical connection between the slides, and to select slides based on the segments grasped by themselves.

Kashihara and Akiyama proposed a model and a cognitive tool of knowledge construction from Web resources that do not often provide a table of contents (Kashihara \& Akiyama, 2013). Referring to this model, in this paper, we have designed a model of learning processes for SBI-learning. The model consists of three phases as shown in Figure 1. In this model, learners are expected to repeat investigating and understanding 
slides under these phases until they finish constructing knowledge satisfied to their viewpoint for learning. In the above three phases, it is very important to find out slides necessary for learning in the first phase because they will finish learning with incomplete knowledge in the subsequent phases if they cannot find out the slides. In the following section, we will accordingly focus on the slides investigation phase.

\subsection{Problem Addressed}

As Kohlhase (Kohlhase, 2007) states, "PowerPoint is a tool that optimizes the creation of graphical slides", P-document tools such as PowerPoint and Keynote seem to focus on supporting the creation of individual slides. On the other hand, there are few tools that consider the relationship between segments and slides, and that provide only limited functionality, such as outline view by slide title and adding sections. Therefore P-documents created using these tools tend not to explicitly represent the roles of each slide and the logical connection between slides. Hence, when learners investigate slides from these P-documents, they need to identify whether the role of each slide represents "the main topic" or "supplementary of the main topic", and whether there are logical connections between slides such as causal or inclusion relationship while they grasp the content of each slide in detail and the structure of the P-documents. However, it is very complicated and difficult for the learners to identify the connections and the roles of slides, and to select slides from the P-document while overviewing the contents of all dozens of slides. As a result, it requires a lot of time for slides investigation.

\section{SUPPORTING TO INVESTIGATING SLIDES}

As described in 2.2 section, it is necessary to concurrently understand the content of the slides and the structure between the slides in SBI-learning, in which the cognitive load would be quite high. Following Mayer's cognitive theory of multimedia learning (Mayer, 2004), we accordingly propose a visualization that enables learners to understand the structure between slides with an appropriate cognitive load.

The proposed visualization allows learners to overview the roles of slides (explanation of the main topic, supplementary explanation of the main topic) and the logical connections between slides (such as causal and inclusive relations). These roles and connections are provided to the learners as a map called slidemap so that they can seamlessly grasp the contents and the boundaries of the segment, and select slides necessary for learning.

\subsection{Visualization of the Roles of Slides}

As described above, slides in a P-document have two roles. The role of "supplementary explanation of the main topic" can be also divided into two types: One is to present the contents that support the main topic (support explanation), and the other is to present the contents that rebut the main topic (rebuttal explanation)". In the slidemap, these roles are visualized as shown in Figure 2 (a).
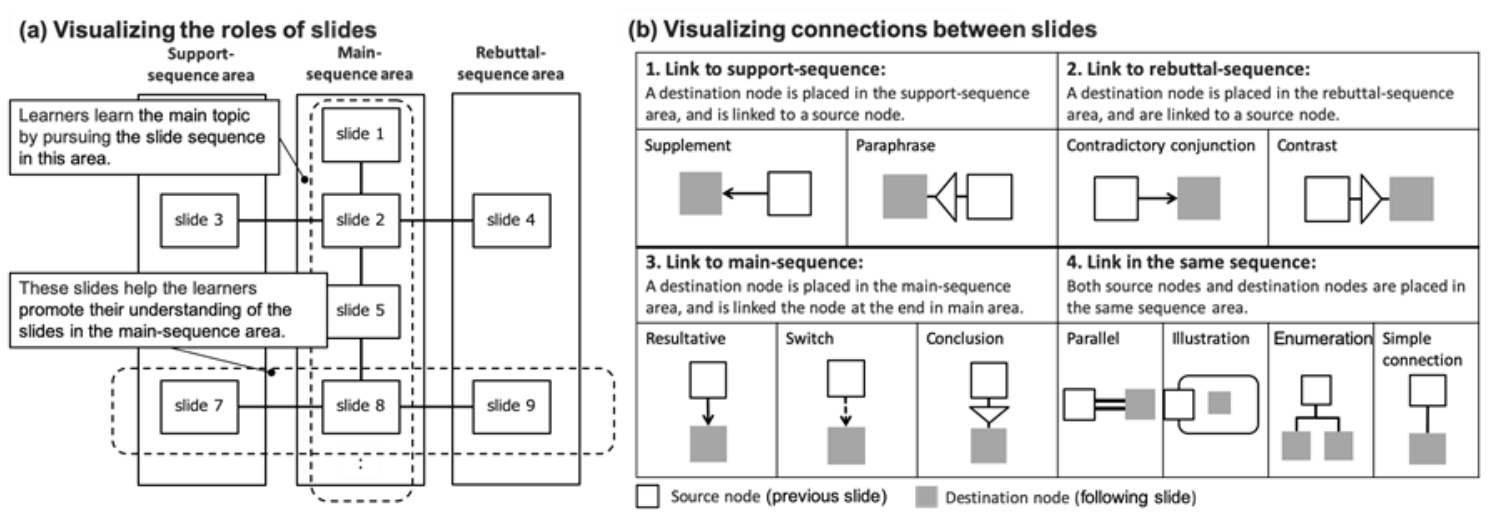

Figure 2. An example of visualization the roles of the slides and the logical connections 
This map consists of three areas, which are main-sequence area, support-sequence area and rebuttal-sequence area. Each slide is also represented as node and the connection between slides is represented as link between the corresponding nodes, as shown in Figure 2 (a). The main-sequence area includes the slides, which describe the main topic. The learners are allowed to pursue the slide sequence in this area to learn the main topic of the P-document. The support-sequence area also includes the slides, which describe the contents supporting the main topic for the slides in the main-sequence area. On the other hand, the rebuttal-sequence area includes the slides, which describe the rebuttal of the main topic in the main-sequence area. The slides in the support-sequence and rebuttal-sequence areas are useful for them to promote their understanding of the slides in the main-sequence area.

\subsection{Visualization of Connections between Slides}

In order to represent the logical connections between the adjacent slides, this paper adopts the functions of conjunctions. The functions of conjunctions allow us to represent the logical connections independent of the slide contents. In this work, we follow the classification of conjunctions in Japanese by Ishiguro (Ishiguro, 2006) to define 10 types of logical connections. Moreover, the connection between the adjacent slides beyond the functions of conjunctions is defined as simple connection. These 11 connection types are visualized as shown in Figure 2 (b), which allow learners to grasp the structure embedded in a P-document in a shorter time. For example, if there is an "Illustration" relationship between two slides, it implies that the including slide represents "a certain content" and the included one represents "a specific example". This allows learners to grasp the relationship of the two slides.

Links representing the logical connections are changed according to the conjunction types as shown in Figure 2 (b). There are four categories of link representations, and the placement area of the destination node is decided according to the category. In addition, segments are not explicitly represented in the slidemap. Learners are expected to become aware of the boundaries of the segments by means of the link representation in the map.

\subsection{Visualizer Tool for Investigating Slides}

This tool is implemented as an add-in for Microsoft PowerPoint 2013, and runs standalone in the PowerPoint on a Windows PC. This tool provides learners with the map representation displaying together on the normal user interface of PowerPoint in order to support the slide investigation.

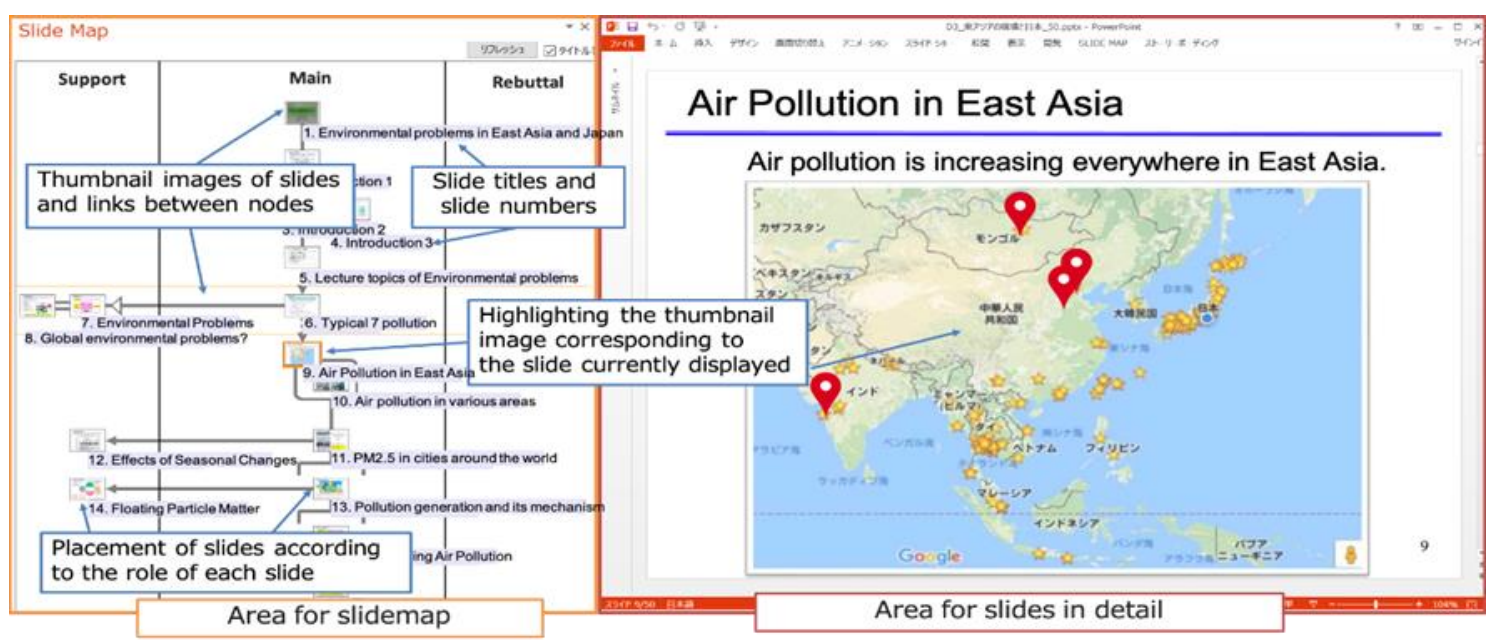

Figure 3. An overview of the proposed tool

As shown in the left of Figure 3, the slidemap displays the structure of the P-Document with the thumbnail images of each slide as nodes and the connections between slides as links. The thumbnail images of slides are placed in the area for slidemap, and the slide numbers and slide titles are displayed near the corresponding thumbnail images. These representations allow learners to overview each slide without confirming the details 
of each slide. The thumbnail image in the map is highlighted corresponding to the slide currently displayed in the area for slides on the right of Figure 3. This feature allows the learners to visually confirm the conjunctive relationship between a slide which they confirm in detail and the ones surrounding it. Wang and Sumiya proposed a quick browsing tool that generates a word cloud based on the texts in a slide to help learners understand the content of each slide (Wang \& Sumiya, 2013). It aims not to grasp the segments but to overview the contents of each slide.

\section{CASE STUDY}

We conducted a case study with 16 participants to confirm whether the proposed tool was effective in the slide investigation phase of SBI-learning. In this case study, we evaluated the differences in learning time and test results between the two conditions, which were slide investigation with normal PowerPoint user interface (referred to as PowerPoint-UI) and slide investigation with the proposed tool (referred to as Slidemap-UI).

\subsection{Preparation}

The participants were 16 undergraduate and graduate students of information engineering ( 3 rd year to 2 nd year of master's degree; male: 12 , female: 4 ; mean age: $22.7 \pm 1.2$ ). The P-documents used in this case study were two documents obtained from SlideShare (LinkdeIn, 2016). One was a document on renewable energy (referred to as Doc 1), and the other was a document on zoology (referred to as Doc 2). Two conditions were set: one for learning Doc 1 with PowerPoint-UI (referred to as "C-Cond"), and the other for learning Doc 2 with Slidemap-UI (referred to as "P-Cond"). The size of the slides displayed in both UIs was the same, and the participants in C-Cond were instructed slide investigation using a thumbnail view of PowerPoint-UI, in which thumbnail images lined up in a row.

The number of slides in each document was 55 slides, the number of segments included in each document was 6 segments, and the average number of slides in a segment was 9.2 slides. The number of slides in each sequence area was almost the same. We also confirmed that prior knowledge about the contents each participant had was almost the same by means of 5-point Likert scale questionnaire. As a result, we believed that the difference of P-documents has little impact on the learning results.

We prepared viewpoints for learning in the experiment, and the participants were instructed to investigate slides related to the viewpoints provided, and to understand the details of the slides. Table 1 shows two viewpoints provided with the participants in each condition. Each condition had two sessions. In Session 1, the participants were given the first viewpoint, and then were required to investigate and learn slides. In Session 2 , they were given the second viewpoint, and then were required to conduct investigative learning. In other words, we conducted a within-participants design, and required each participant to do two sessions under each condition. The reason for conducting two sessions under one condition was to evaluate the effectiveness of the slide map under the first use of P-document and the second use of the same P-document with some knowledge of the structure.

Table 1. The viewpoints provided with the participants in each session

\begin{tabular}{lll}
\hline First session & Second session \\
\hline C-Cond & $\begin{array}{l}\text { The environmental loads compared to } \\
\text { depletable energy }\end{array}$ & $\begin{array}{l}\text { The problems in spreading the use of solar } \\
\text { power in Japan }\end{array}$ \\
\multirow{2}{*}{ P-Cond } & $\begin{array}{l}\text { The layout of exhibition of great academic } \\
\text { significance in zoos }\end{array}$ & $\begin{array}{l}\text { About animals that can only exhibit their herd } \\
\text { in females }\end{array}$ \\
\hline
\end{tabular}

\subsection{Procedure}

First, the participants practiced the operation of each UI with a sample P-document that was different from Doc1 or Doc2 until they had no unclear point in using it. The experimenter next instructed the participants as follows: (1) You were required to investigate and learn slides according to the viewpoint provided using the UI specified by the experimenter. The learning time was within 20 minutes in one session. If you finished 
learning earlier than 20 minutes, you were also required to tell the experimenter when you finished, (2) After learning in one session, you were required to answer an understanding test about the viewpoint provided, (3) After learning in one condition, you were required to answer a questionnaire about the System Usability Scale (SUS) (Brooke, 1996) of UI that you used, and (4) you were finally required to answer the five Likert scales of questionnaire about the subjective usability of each UI as shown in Table 2.

Table 2. Each question item of subjective questionnaire

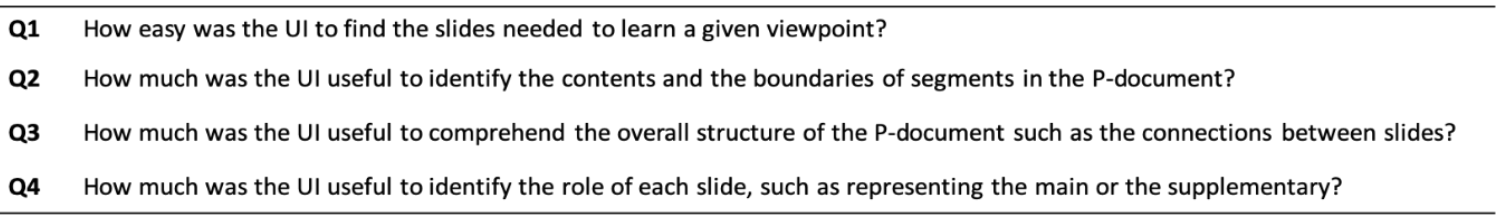

In this study, we also recorded the learning time and the viewing frequency of each slide by each participant as quantitative metrics to evaluate the effectiveness of the slide investigation, which were captured by the logger implemented in each UI. The hypotheses we set up in this study were as follows:

H1: The Slidemap UI encourages learners to investigate the slides related to the viewpoint in a shorter time than the PowerPoint-UI,

H2: The Slidemap UI encourages learners to more intensively investigate the slides that are suitable for understanding the viewpoint than the PowerPoint-UI and

H3: The Slidemap UI has higher usability for investigating the slides than the PowerPoint-UI.

\subsection{Results}

The results were analyzed by Wilcoxon signed-rank test for each session or each condition, the symbols in the graphs represent significant differences at $\dagger: \mathrm{p}<.10, *: \mathrm{p}<.05, * *: \mathrm{p}<.01$ and $\mathrm{r}$ represents effect size $(0.1<=\mathrm{r}<0.3$ : small, $0.3<\mathrm{r}<=0.5$ : medium, $\mathrm{r}>0.5$ : large $)$ respectively.

\subsubsection{Learning Time and Viewing Frequency}

Figure 4 (a) shows the average of the total learning time in each session. In the first session of each condition, the average time in $\mathrm{P}$-Cond was about one minute shorter than C-Cond, but there was no significant difference $(\mathrm{Z}=-1.24, \mathrm{p}=0.21, \mathrm{r}=0.31$.) In the second session of each condition, however, the average time in $\mathrm{P}-\mathrm{Cond}$ was about the half of the average time in C-Cond, and there was a significant difference $(Z=-3.46, p<.01$, $\mathrm{r}=0.87$.)

Figure 4 (b) shows the ratio of the average learning time of the slides related to the viewpoint to the time of all slides in each session. If this ratio is higher, it suggests that the participants spent more time for learning the slides related to the viewpoint. In the first session of each condition, the ratios in C-Cond and P-Cond were $21.8 \%$ and $35.9 \%$ respectively. In the second session of each condition, the ratios in C-Cond and P-Cond were also $59.1 \%$ and $82.1 \%$. There was no significant difference in the first session and was significant difference in the second session between P-Cond and C-Cond (in the first session: $\mathrm{Z}=1.24, \mathrm{p}=.21, \mathrm{r}=0.31$, in the second session: $\mathrm{Z}=-2.89, \mathrm{p}<.01 \mathrm{r}=0.72$.)

Figure 4 (c) shows the ratio of the average viewing frequency of the slides related to the viewpoint to the frequency of all slides in each session. If this ratio is higher, it suggests that the participants more repeatedly view the slides related to the viewpoint. In the first session of each condition, the ratios in C-Cond and P-Cond were $20.6 \%$ and $15.5 \%$, and there was no significant difference $(Z=-1.29, p=.20, r=0.32$.) In the second session of each condition, the ratios in C-Cond and P-Cond were $30.8 \%$ and $56.2 \%$, and there was a significant difference $(Z=-2.84, \mathrm{p}<.01, \mathrm{r}=0.71$.

We also compared the average of the viewing frequency of all slides in C-Cond with the one in P-Cond. In the first session, the frequency in C-Cond was 82.1 times and the one in P-Cond was 93.6 times. This result suggests that the participants tended to overview more slides in the P-document. In the second session, the frequency in C-Cond was 51.6 times and P-Cond was 26.5 times. This result suggests that the participants more intensively investigate the slides related to the viewpoint based on some knowledge about the structure of the P-document grasped in the first session (Due to the space limitation, the graph is not shown here.) 
(a) The average of the total learning time

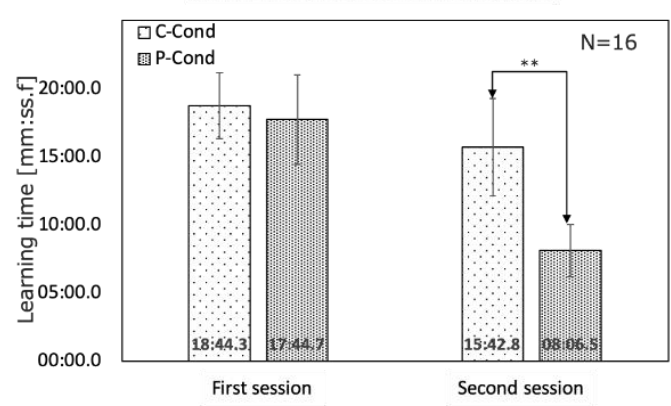

(c) The ratio of the average viewing frequency of the slides related to the viewpoint to all slides

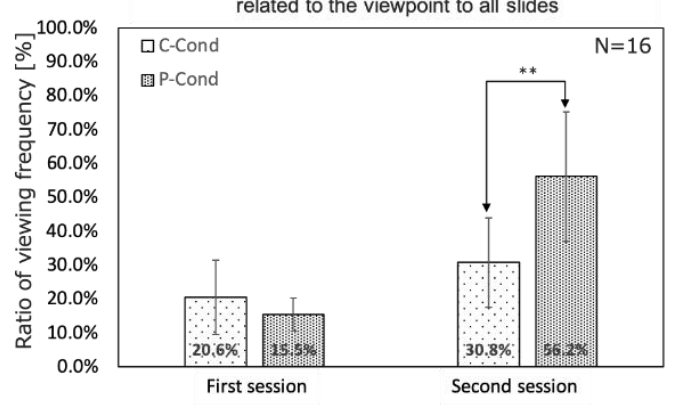

(e) The average score of the SUS questionnaire

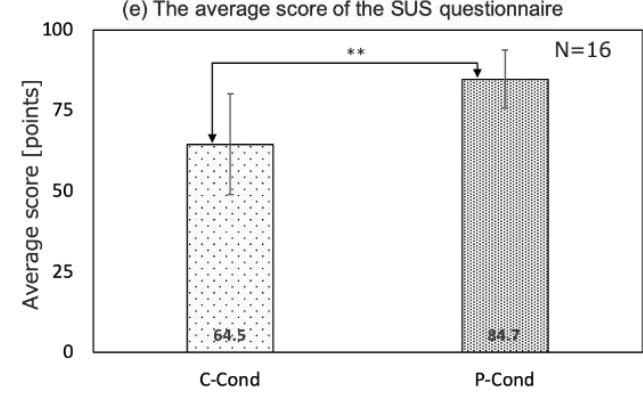

(b) The ratio of the average learning time of the slides

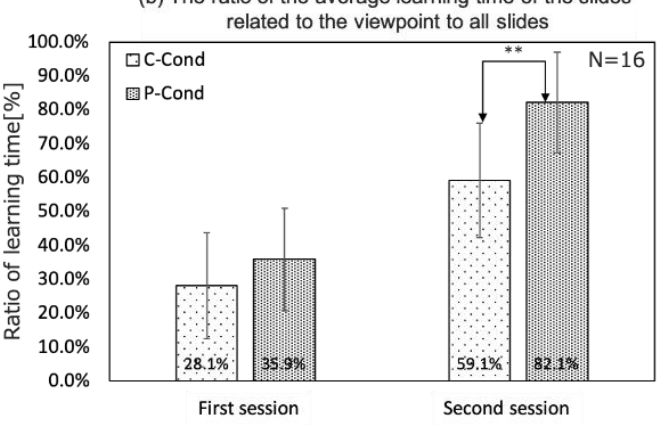

(d) The average score of the understanding test
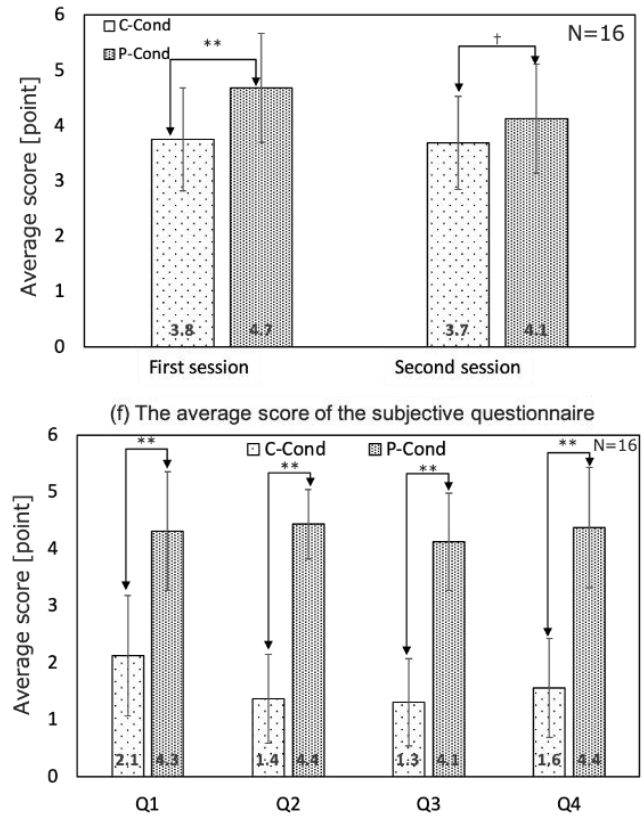

Figure 4. Results of the case study

\subsubsection{Understanding Test}

The understanding test consisted of five questions about the contents of the slides, each of which was given one point, for a total of five points. When a question is answered with multiple elements, the score is divided by one. For example, if a question is answered with two elements, the score is 0.5 . Figure 4 (d) shows the average scores of the test for all the participants in each condition. In the first session, the average scores of understanding test in C-Cond and P-Cond were 3.8 points and 4.7 points respectively. In the second session, the average score in C-Cond and P-Cond were 3.7 points and 4.1 points. There was a significant difference in the first session and was a tendency of significant difference in the second session between P-Cond and C-Cond (in the first session: $Z=-3.01, p<.01 \mathrm{r}=0.75$, in the second session: $Z=-1.67, \mathrm{p}<.10, \mathrm{r}=0.42$.)

\subsubsection{Usability of User Interface}

Figure 4 (e) shows the average scores of the SUS questionnaire for all participants in each condition. The average scores in C-Cond and P-Cond were 64.5 points and 84.7 points, and there was a significant difference. SUS is the metrics to evaluate the usability of information systems, and the range of score is from 0 to 100 . If the score is closer to 100, it indicates a higher usability. P-Cond accordingly makes an impression of a good UI to the participants. Figure 4 (f) shows the results of the subjective questionnaire for each condition. For all questions from Q1 to Q4, the average score in P-Cond was higher than the one in C-Cond, with significant 
differences $(\mathrm{Q} 1: \mathrm{Z}=-3.05 . \mathrm{p}<.01 \mathrm{r}=0.76, \mathrm{Q} 2: \mathrm{Z}=-3.24, \mathrm{p}<.01 \mathrm{r}=0.81, \mathrm{Q} 3: \mathrm{Z}=-3.53, \mathrm{p}<.01 \mathrm{r}=0.88$, Q4: $\mathrm{Z}=-3.49, \mathrm{p}<.01, \mathrm{r}=0.87)$.

Following the results described above, we confirm whether the hypotheses H1, H2, and H3 are valid. From the average of total learning time in one session (Figure 4 (a)) and the scores of the understanding test (Figure 4 (d)), the Slidemap-UI first allowed the participants to finish learning with a shorter time, and to get higher scores in the understanding test than the PowerPoint-UI not only in the first session but also in the second session. These results support H1. From the ratio of the average learning time (Figure 4 (b)), next, the Slidemap-UI can be more effective than the PowerPoint-UI. From the ratio of the average viewing frequency of the slides (Figure 4 (c)), when the participants have some knowledge about the structure of P-document, the Slidemap-UI allowed the participants to learn more intensively the slides in the second session than in the first session. These results suggest H2. Finally, the SUS score (Figure 4 (e)) and the subjective questionnaire (Figure 4 (f)) for each condition suggest that the Slidemap-UI has higher usability and is subjectively more suitable for supporting investigating slides in SBI-learning than the PowerPoint-UI. These results support H3.

\section{CONCLUSION}

In this paper, we proposed supporting slides investigation with the slidemap that visualizes the logical connections between slides based on the 11 types of relationships corresponding to the meanings of Japanese conjunctions. We also conducted a case study with 16 participants whose purpose was to confirm the effectiveness of the tool for slides investigation. As a result, the proposed Slidemap-UI allowed the participants to finish learning with a shorter time and higher understanding scores than the PowerPoint-UI. In addition, the proposed UI encouraged the participants intensively to learn the slides related to the viewpoint with higher usability. In future, we will study the algorithm for automatic generation of the proposed slide map, design the UI for knowledge construction and the UI for slides investigation in multiple P-documents.

\section{ACKNOWLEDGEMENT}

The work is supported in part by JSPS KAKENHI Grant Number JP17H01992.

\section{REFERENCES}

Brooke, J., 1996. SUS: A "quick and dirty" usability scale. Usability. Evaluation in Industry, pp. 189-194.

Corbeil, G., 2007. Can PowerPoint Presentations Effectively Replace Textbooks and Blackboards for Teaching Grammar? Do Students Find Them an Effective Learning Tool? CALICO Journal, 24(3), pp.631-656.

Ishiguro, K., 2006. Sentences are connected with conjunction (in Japanese). Koubunsha, Tokyo, JAPAN.

Kashihara, A. and Akiyama, N., 2013. Learner-created scenario for investigative learning with web resources. Proceedings of the 16th International Conference on Artificial Intelligence in Education (AIED2013), LNAI7926, pp.700-703.

Kohlhase, A., 2007. Semantic PowerPoint: Content and Semantic Technology for Educational Added-Value Services in MS PowerPoint. Proceeding. of World Conference on Educational Multimedia, Hypermedia \& Telecommunications (ED-MEDIA2007). Vancouver, Canada, pp. 3576-3583.

LinkedIn Corporation, SlideShare, viewed 20 April 2016, <http://www.slideshare.net/>.

Mayer, R. E., 2014. Cognitive theory of multimedia learning. The Cambridge handbook of multimedia learning. Cambridge University Press, pp. 43-71.

Shibata, Y. et al, 2013. Skill transfer from learning to creating presentation documents. Proceedings of the 12th Information Technology Based Higher Education and Training (ITHET2013). Antalya, Turkey, pp. 1-6.

Wang, Y., and Sumiya, K., 2013. Dynamic Word Clouds: Context-based Word Clouds of Presentation Slides for Quick Browsing. Proceedings. of 6th IIMSS2013. Sesimbra, Portugal, pp. 108-117. 